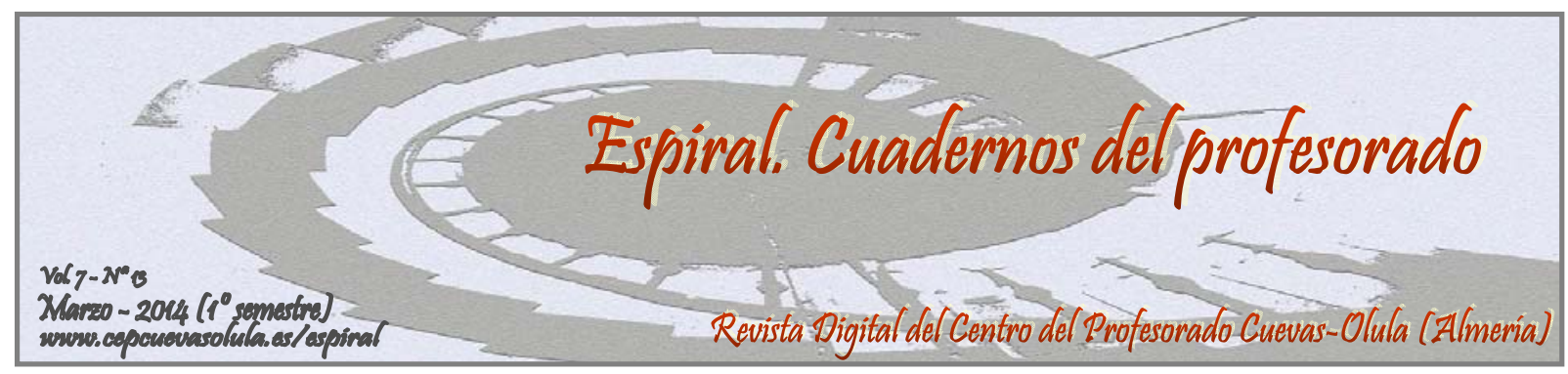

\title{
TRABAJANDO LAS COMPETENCIAS EN ENSEÑANZA SECUNDARIA OBLIGATORIA DESDE LA OBRA CLÁSICA LÁZARO DE TORMES
}

\author{
WORKING ON KEY SKILLS IN COMPULSORY SECONDARY EDUCATION \\ FROM THE CLASSIC FICTION WORK LÁZARO DE TORMES
}

\section{Lidia Irene Olmos Moreno}

Departamento de Lengua Castellana y Literatura, IES Puerta de Arenas, Jaén, España

RESUMEN: Tomando como eje una obra de lectura obligada como es Lázaro de Tormes, se muestra cómo pueden plantearse diversas tareas para acceder a las ocho competencias básicas en Enseñanza Secundaria Obligatoria (ESO) y, lo más importante, haciéndolo de forma que el paso de una tarea a otra no suponga una ruptura entre ambas. Lo que se pretende es que el alumnado entienda que todas las destrezas que se le piden son necesarias y que, desde cualquier materia pueden trabajarse cuestiones, a priori, distantes y relegadas, hasta ahora, a cada una de las distintas materias que integran el currículo. Desde la materia de Lengua Castellana y Literatura se ofrece una experiencia que muestra cómo pueden realizarse tareas relacionadas con dichas competencias, recogiéndose contenidos relacionados con Ciencia, Arte, Historia, educación en valores, razonamiento matemático, expresión verbal, etc. logrando en el alumno un aprendizaje global, que le permita desenvolverse en diferentes ámbitos de la vida académica, personal y, en última instancia, laboral.

Palabras clave: competencias, destrezas, autonomía, aprendizaje trasversal.

\section{ABSTRACT: Abstract}

Departing from a set reading such as Lázaro de Tormes, it is demonstrated how a range of different activities can be designed to work on the eight key skills competences in General Compulsory Secondary Education (GCSE). Most importantly, the transition from one task to another does not involve a break between them. The goal is that students understand that all the skills that are requested are necessary and that, from any subjet we can work on issues a priori, remote and neglected, until now, to each of the subjects that shape the curriculum. In the area Spanish Language and Literature, tasks related to the eight competences can be developed as they offer content related to science, art, history, citizenship, mathematical reasoning, verbal expression, etc., making students achieve a global, learning and enabling them to function in different areas of academic, personal life and, ultimately, employment.

Key words: key competences, skills, autonomy, crosscurricular topics.

Olmos Moreno, L. I. (2014). Trabajando las competencias en Enseñanza Secundaria Obligatoria desde la obra clásica Lázaro de Tormes. Espiral. Cuadernos del Profesorado, 7(13), 51-58. Disponible en: http://www.cepcuevasolula.es/espiral.

Fecha de recepción: 06/07/2013

Fecha de aceptación: 10/01/2014
Enviar correspondencia a: profelidia.lengua@gmail.com 


\section{1.- INTRODUCCIÓN}

El sistema educativo español, en un intento de igualarse a la gran mayoría de sistemas educativos europeos, recoge, desde el año 2006 (Ley Orgánica de Educación, de 3 de mayo, LOE) una serie de medidas encaminadas a lograr el "desarrollo integral de la persona". Entre ellas encontramos el hecho de que el objetivo último al terminar la Enseñanza Secundaria Obligatoria es que el alumnado domine lo que ha venido en llamarse competencias básicas, entendidas como un conjunto de destrezas interiorizadas por el alumno/a que le permiten saber aplicar los conocimientos adquiridos y desenvolverse en diferentes contextos de la vida académica, personal y, en última instancia, laboral.

Pensadores y lingüistas de todos los tiempos como Habermas (1987), Jakobson (1984), Saussure (1991), Chomsky (1979) o Coseriu (1967), entre otros, han analizado la competencia lingüística, (llamada competencia comunicativa, argumentativa, discursiva, textual...) valorándola como el eje del conocimiento, como la llave que abre la puerta al conocimiento, sea de la índole que sea.

En esta misma línea, Gómez y Arcos (2007, p.1) consideran que el eje vertebrador de todas las competencias que ha de desarrollar el alumnado es la competencia lingüística, “entendida como un elemento básico fundamental para la adquisición de nuevos aprendizajes y para su desarrollo personal". Además, sostienen que "el perfeccionamiento de esta competencia no puede ser en absoluto tarea exclusiva del profesor de Lengua" sino que ha de abordarse desde cualquier materia. Por este motivo, se hace necesario trabajar la competencia lingüística desde cualquier área o materia.

Detenerse en leer con el alumnado de forma comprensiva, con buena entonación; corregir las faltas de ortografía, incrementar el caudal léxico, trabajar la expresión coherente, tanto de forma oral como escrita, no han de ser contenidos que se releguen únicamente a las clases de Lengua. Es cierto que todo docente tiene un programa que cumplir, unos contenidos que enseñar pero los mencionados más arriba son imprescindibles para que el alumno acceda a cualquier conocimiento nuevo, lo interiorice, lo procese, lo asimile y lo haga suyo. Lo novedoso ahora es integrar esas destrezas en cada una de las materias que integran el currículo en Enseñanza Secundaria Obligatoria.

Una manera de lograr que las distintas destrezas se trabajen de forma integrada es utilizando como vehículo las "tareas integradas" que podemos definir como "una secuencia didáctica organizada de tal forma que ayude a los estudiantes a lograr la realización de una actividad compleja relacionada con distintas áreas de conocimiento y con la experiencia vital de los propios estudiantes" (Trujillo, 2010).

Es aquí donde la competencia lingüística adquiere una importancia notoria ya que implica no sólo saber expresar y comprender correctamente mensajes orales y escritos sino que también es esa destreza que hace posible a la persona asimilar una información, estructurarla, discriminar lo esencial de lo superfluo, analizarla de forma crítica, expresando la propia opinión, etc. Es debido a esa funcionalidad por lo que debe abordarse desde todas las materias y, para ello, debemos dar cabida a medidas como las que aquí se proponen, donde, en todo momento, se trabaja la competencia lingüística y, de forma hilada y recurrente, el resto de las competencias establecidas legalmente.

Las actividades que aquí se presentan se llevaron a cabo en el contexto de un grupo de veinte alumnos/as de $3^{\circ}$ de ESO, pertenecientes a un IES de la provincia de Almería. El objetivo de dar a conocer esta experiencia es dotar al docente de nuevas herramientas e ideas para contribuir, desde cualquier materia, al desarrollo de las ocho competencias básicas que establece la actual legislación educativa, tomando como eje la competencia lingüística.

Con tareas como las que se presentan, el alumnado se ejercita en la expresión/comprensión verbal, el razonamiento matemático, contenidos relacionados con la Ciencia, el Arte, la ciudadanía, etc. y todo ello favoreciendo un aprendizaje funcional y autónomo unas veces, o colaborativo otras, pero logrando siempre que dicho aprendizaje sea entendido por el alumnado como algo significativo, lógico, consiguiendo así una motivación mayor por su parte y desarrollando unas capacidades que le permiten desenvolverse en contextos diferentes y ante problemas diversos (Martín y Coll, 2003). 


\section{Origen de la experiencia}

Desde que, como ya se ha expuesto anteriormente, la nueva legislación educativa (LOE) contempla que el alumnado de Enseñanza Secundaria Obligatoria ha de desarrollar una serie de competencias que deben trabajarse desde todas las materias, se le plantea al docente una incógnita: ¿Cómo trabajar desde mi área, desde mi especialidad, contenidos y destrezas vinculados a otras áreas? Una premisa queda clara desde el principio: las competencias no pueden "enseñarse” de forma teórica, sino que han de lograrse desde un enfoque práctico y funcional.

Hay competencias que se prestan a que puedan ser abordadas desde cualquier materia (como hemos apuntado con anterioridad, la lingüística, por ejemplo, con el simple hecho de hacer que el alumnado lea comprensivamente un enunciado, la teoría de la unidad; pidiéndole la realización de trabajos de investigación en los que debe recoger, por escrito, la información que se le pide, conclusiones, etc., corrigiéndole errores ortográficos, etc.) pero hay otras competencias que resulta más complejo trabajar desde cualquier materia (la competencia para el conocimiento e interacción con el mundo físico o la competencia matemática, por ejemplo, no se relacionan de una forma directa con la lingüística o la cultural, y no es sencillo trabajarlas desde áreas con la Lengua, Historia, etc.).

A ello se añade que, como es obvio, lo ideal sería abordar las competencias, desde cualquier materia, pero sin que ello suponga una ruptura con los contenidos que se estén trabajando en cada momento. Es esta una preocupación latente en el docente. Así, surgen trabajos en los que se reflexiona sobre qué aspectos o contenidos quedan integrados en cada una de las competencias, de manera que, de una forma u otra, pueda encontrarse relación entre contenidos pertenecientes a las distintas competencias. Un buen ejemplo es la tabla de descriptores elaborada por el Servicio de Inspección de las Palmas con la colaboración del Centro del Profesorado (CEP) de Telde, que pueden ayudarnos a elegir contenidos pertenecientes a las diferentes competencias para que, como ya se ha dicho, se consiga un aprendizaje integrado de todas ellas.

Ante esta preocupación por abordar las ocho competencias de forma coherente e hilada, surgió la idea de utilizar una obra clásica Lázaro de Tormes, de lectura obligada para nuestros jóvenes, que, además, suele tener bastante éxito entre el alumnado, como eje vertebrador de una serie de actividades con las que se conseguía trabajar dichas competencias.

Como docente de Lengua Castellana y Literatura, el principal objetivo era fomentar la lectura, el interés del alumnado por la obra que se le había pedido que leyera de forma obligatoria durante el primer trimestre. La lectura estimula la imaginación y ayuda al desarrollo del pensamiento abstracto, favorece la correcta expresión, abre la mente, etc. (Martínez, 2009). En la actual sociedad de la comunicación, caracterizada por la sobreabundancia de datos, la lectura comprensiva tiene un papel clave para convertir la información en conocimiento. Así, durante dicho primer trimestre, el alumnado leía en casa una serie de páginas que luego eran comentadas, releídas y debatidas, si se daba el caso, durante una hora de clase a la semana. A raíz de las interminables preguntas que alumnos y alumnas formulaban sobre los lugares en los que se desarrolla la obra, la época, los acontecimientos que se citan, etc. se hacía evidente que contábamos con la herramienta ideal que todo docente anhela: el entusiasmo de su alumnado. De esta manera, se animó a algún alumno al que se le daba bien el dibujo a que ilustrara algunos ambientes o personajes que se describían en la obra (trabajando, de alguna manera, la competencia artística), a otros se les pidió que investigaran son los acontecimientos bélicos a los que se hace referencia (competencia social y ciudadana),a aquellos que amaban la literatura y el relato se les sugirió que imaginasen las cartas que Lázaro escribiría a su madre contándole los avatares de su existencia (competencia lingüística)... Prácticamente de forma inconsciente, el alumnado estaba trabajando varias competencias de forma entretenida y amena y se tomó entonces la decisión de buscar la manera de trabajarlas todas, a través de otras actividades siempre relacionadas con el libro.

\section{Objetivos}

- Compartir una iniciativa que permite trabajar las ocho competencias básicas desde una perspectiva cohesionada e integradora.

- Aportar ideas al docente para que pueda desarrollar y promover el hábito lector, entendiendo la lectura como fuente de diversión y entretenimiento, como alternativa que 
tener en cuenta en el tiempo de ocio.

- Hacer hincapié en la necesidad de conseguir que el alumnado adquiera una capacidad reflexiva, crítica y selectiva ante el amplio volumen de información al que pueden acceder en la actualidad.

\section{2.- METODOLOGÍA}

Partiendo de la premisa de que la prioridad de estas actividades reside en desarrollar las ocho competencias básicas entre las que se encuentra la competencia de aprender a aprender y la de la autonomía e iniciativa personal, no cabe contemplar otra metodología que no sea la que entienda al alumno como eje y motor del proceso de enseñanza/aprendizaje. La figura del profesor adquiere un segundo plano, limitándose a ser mero conductor del proceso, haciendo correcciones, dando indicaciones, colaborando y ofreciendo ayuda para conseguir que el alumnado llegue al fin propuesto. Además, tendremos siempre en cuenta el conocimiento previo y las experiencias del alumnado con objeto de facilitar el aprendizaje significativo (el alumno aprende mejor aquello que entiende como algo lógico y que puede relacionar con su propia experiencia, entorno, etc.).

Dependiendo de la tarea que se realice, se trabajará en parejas (aprendizaje colaborativo) o de forma individual (aprendizaje autónomo); se utilizarán materiales tradicionales (enciclopedias, cuaderno) o las nuevas tecnologías (internet, enciclopedias digitales, blogs, procesador de textos, etc.). Se fomentará, siempre que sea posible, la actitud indagadora y analítica en el alumnado. Esta ha de ser la tónica común en la mayoría de las tareas pues, de esta forma, el alumnado aprende realmente, se implica en su propio aprendizaje, porque adopta un papel eminentemente activo en él (Estaire, 2004).

Debido a que en el aula encontrábamos varios niveles (alumnado con distintos ritmos de aprendizaje), entendimos que sería necesario elaborar actividades que dieran respuesta a sus necesidades, su propio ritmo, su nivel de aptitud. De esta manera, en las actividades que se detallan a continuación, se distinguen actividades comunes (dentro de las cuales se contemplan distintos niveles de complejidad), que todo el grupo realizaba y actividades de ampliación, pensadas para algunos alumnos/as de aprendizaje más rápido.

En cuanto a la temporalización, estas actividades ocuparían una hora a la semana, durante un trimestre (unas 12 horas).

Para estas actividades, utilizamos la obra Lazaro de Tormes de la editorial Vicens Vives.

\section{Actividades encaminadas al logro de las distintas competencias}

\section{a) Competencia lingüística}

\section{Comunes}

- (Individual) Escribe un texto de unas quince líneas donde resumas el argumento del libro

- (Individual) Imagina y escribe la carta que pudo escribir Lázaro a su madre cuando abandonó al ciego.

- (Individual) Cuenta qué episodio del libro te ha gustado más, qué personaje, etc. ¿Cambiarías algo de la historia?

- (Individual) Busca en el diccionario virtual o impreso de la Real Academia Española (DRAE), el significado de las siguientes palabras que aparecen en el libro: argolla, tolondrón, asir, mezquino, oblada, lóbrega, alfámar, mancilla, holgar, birrete, bonete, almoneda, medrar, bula.

- (Por parejas) Propón un sinónimo para cada una de las siguientes palabras. Puedes buscarlas en la página en la que aparecen para entender su significado contextual. Si no entiendes su significado, búscalo en el diccionario: ventura (3), preñada (7), bestias (9), hurtaba (11), padeciendo (12), simpleza (13), fardel (17), desamparaba (20), determiné (32), toparon (37).

\section{Ampliación}

(Individual) Se ha especulado mucho sobre quién puede ser el autor de esta obra. Busca información en Internet al respecto y refleja alguna de estas teorías. 


\section{b) Competencia matemática}

\section{Comunes}

- (Por parejas) Elabora una gráfica (cronograma) en la que recojas la temperatura que se da en Toledo a lo largo del año.

- (Por parejas) ¿Cuál es la temperatura media en Toledo, tanto en verano como en invierno?

- (Individual) Si la obra se publica en 1499, ¿cuántos años hace?

nació?

- (Individual) Imaginemos que Lázaro tenía 21 años cuando se publicó la obra, ¿en qué año

- (Individual) Busca en Internet la moneda que se usaba en la época y a cuánto ascendía el sueldo de un trabajador.

\section{Ampliación}

- (Individual) Imagina lo que compraban los españoles de entonces para comer. Intenta elaborar una lista de alimentos y propón un precio aproximado (ten en cuenta la información obtenida en el ejercicio anterior).

\section{c) Competencia cultural y artística}

\section{Comunes}

- (Por parejas) Busca información sobre el estilo arquitectónico de la época. Elabora un breve texto expositivo con las principales características. estilo.

- (Por parejas) Elabora un dibujo a lápiz de algún edificio con las características de dicho

- (Por parejas) Realiza un texto expositivo explicando las características de la pintura del ejercicio anterior, atendiendo al estilo.

- (Por parejas) Busca en internet imágenes sobre algunos edificios citados en el texto (catedrales, plazas, puentes, etc.) y expón las características de este tipo de arquitectura.

- (Individual) Busca información sobre otras novelas (españolas o europeas) que cuenten con un pícaro como protagonista.

\section{Ampliación}

- (Individual) Busca en internet la pintura que un artista español muy conocido realizó inspirándose en un pícaro. Cópiala en un documento de texto y realiza una descripción del muchacho. ¿Coincide con la imagen mental que tenías de Lázaro? Justifica tu respuesta.

\section{d) Competencia social y ciudadana}

\section{Comunes}

- (Por parejas) Busca información sobre la importancia de la religión en aquella época en la vida política. Elabora una pequeña reflexión sobre la calidad humana de los personajes relacionados con la iglesia que aparecen en la obra.

- (Individual) En el texto, Lázaro pregona las condenas de los reos. A muchos de ellos los condenaban a galeras. Busca información y explica en qué consistía esa condena.

- (Por parejas) Como hemos visto en la obra, se pregonaban las condenas de los reos. Opina sobre esta práctica.

- (Individual) Busca imágenes que reflejen la moda femenina en el siglo XV. Da tu opinión sobre este tipo de ropa que tapaba, por completo, a la mujer. ¿ Sigue habiendo culturas en las que las mujeres no deben llevar ropa que deje a la vista piernas o escote? ¿Qué opinas al respecto?

- (Individual)Lázaro fue pregonero. En la época era así como se transmitían las noticias, pues no había otros medios de comunicación. Explica qué aspectos positivos y negativos encuentras en la televisión e imagina qué harías en tu tiempo libre si no existiera dicho aparato. Pregunta a tus abuelos si creen que la televisión ha cambiado la manera de divertirse en los jóvenes. 


\section{Ampliación}

- (Individual) El libro se publica a finales del siglo XV. Busca información sobre la situación sociopolítica de España en esos años. Da tu opinión sobre la convivencia de distintas culturas y sobre las diferentes órdenes de expulsión de musulmanes y judíos que se produjeron en aquellos años.

\section{e) Competencia para el conocimiento e interacción con el mundo físico}

\section{Comunes}

- (Individual) A lo largo de la obra hay constantes alusiones al tiempo meteorológico. Busca información sobre el clima en esa zona de España (lluvioso o seco, frío o cálido). ¿A qué tipo de clima pertenece?

- (Por parejas) Busca información sobre la visión que se tenía en la época sobre los planetas y el espacio. Ten en cuenta que aún no había formulado Galileo Galilei su teoría. Habla también de dicha teoría y sus consecuencias.

- (Por parejas) Busca información sobre la esperanza de vida que se tenía en la época en la que se escribe el libro y compárala con la que se tiene en la actualidad. Explica a qué crees que se debe la diferencia.

- (Por parejas) Busca información sobre el censo de población que había en 1500 en España y compáralo con la actualidad. Explica, dando tu opinión, las diferencias.

\section{Ampliación}

- (Por parejas) Infórmate sobre las características de los planetas que forman nuestro sistema solar: extensión, temperatura, distancia con respecto al Sol y a la Tierra... Según estas características, ¿podría haber vida en algún planeta?

\section{f) Competencia digital}

No elaboraremos actividades específicas puesto que, como puede comprobarse, en las actividades dedicadas a las diferentes competencias se requiere el uso del ordenador, internet, páginas web, procesador de textos, correo electrónico (la mayoría de actividades han de remitirse al profesor a través del correo electrónico, como archivo adjunto), etc.

\section{personal}

g) Competencia de aprender a aprender y competencia para la autonomía e iniciativa

De la misma manera que en el caso anterior, con la realización de las distintas actividades se trabaja la autonomía (en las que se realizan de forma individual) y la iniciativa personal, ya que no se les da la página web en la que pueden hallar la información que se les pide; en otras actividades tienen que reflexionar y aportar su opinión, siendo, a veces, críticos.

La de aprender a aprender también se trabaja en todas las actividades, puesto que se requiere que busquen una información concreta que se les pide, extraigan sólo aquellos datos que se requieren, y esto les lleva a tener que resumir, redactar, deducir, etc.

\section{3.- REPERCUSIÓN PEDAGÓGICA}

En muchas ocasiones, compañeros de diversas materias han exteriorizado una preocupación común y frecuente, sobre todo, en el primer ciclo de ESO: "el alumnado no entiende lo que lee, no sabe expresarse, no tiene una competencia léxica aceptable, no es capaz de sintetizar información, de ceñirse al tema, detectar la idea principal... ¿qué puedo hacer?”. Quizá, el principal problema sea que nos obsesionamos por cumplir con el programa, por dar todos los contenidos que recogen los libros de texto, y nos olvidamos de que estamos empezando la casa por el tejado. Deberíamos priorizar contenidos y objetivos. Muchos de ellos tienen continuidad a lo largo de la ESO, otros debemos dejar que sea el alumnado el que acceda a ellos de forma autónoma. Como ya expuse en la introducción, la competencia lingüística, el dominio de destrezas como saber escuchar y leer es la base del conocimiento. Como docentes, debemos elaborar materiales que permitan al alumnado acceder a los 
contenidos relacionados con nuestra materia pero sin olvidarnos de que para que un alumno capte una información concreta, deduzca o sintetice, debemos enseñarlo primero.

Llevar al aula tareas como la que he presentado en este trabajo tiene, a todas luces, efectos positivos en el alumnado, que accede a un corpus de conocimientos de forma más integrada y entiende que cada materia (Lengua, Matemáticas, Ciencias Naturales, Historia, etc.) no es una realidad aislada sino que se compenetran y son necesarias, de forma recíproca, en la adquisición de nuevos conocimientos.

Iniciativas como esta deberían imitarse desde todas las materias, buscando un elemento que sirva como eje de tareas diversas (un texto, un objeto, una historia, una situación, una imagen, etc.) lográndose así que el proceso de aprendizaje de nuestro alumnado sea mucho más rico y funcional. No es tarea sencilla y de sobra es sabido que hay materias que se dan más que otras a realizar actividades como las propuestas pero, en todo caso, es esta una práctica que ha de empezar a trabajarse y todos, docentes y alumnado, hemos de acostumbrarnos a ella, hemos de entender que en estos tiempos se busca la formación integral del alumnado, que no sólo interesan los conocimientos conceptuales, académicos, por llamarlos de alguna manera, sino también otros aprendizajes que enriquecen al alumno/a como persona y lo hacen un ser más válido para desenvolverse en la sociedad.

\section{4.- EVALUACIÓN}

\section{Evaluación del alumnado}

En cada actividad que encomendábamos a nuestros alumnos/as, evaluábamos varios aspectos: interés y esfuerzo, rapidez en la realización, corrección ortográfica y gramatical (en el caso de actividades escritas), concreción temática, madurez crítica (en los casos en los que se pedía la opinión personal), creatividad, etc.

Podemos decir que casi un 75\% del alumnado logró realizar las actividades completamente, encontró las respuestas correctas, realizó valoraciones personales aceptables según su grado de madurez y, con todo ello, trabajó y desarrolló satisfactoriamente las competencias básicas establecidas para ESO.

\section{Mecanismos de evaluación y criterios de calificación}

Partiendo de la base de que dedicamos una hora a la semana a estas actividades y de que trabajan las ocho competencias básicas, se decidió en el Departamento de Lengua Castellana y Literatura que la nota obtenida por cada alumno en esta experiencia supusiera un $20 \%$ de la nota final del trimestre (las pruebas escritas ordinarias tienen un peso del $60 \%$ y el trabajo diario en casa (realización de tareas propuestas diariamente dentro de la materia) un 20\%). Las actividades se evalúan de la siguiente forma:

1- Actividades comunes por parejas (valen dos puntos): la nota obtenida en las actividades se divide entre los miembros de la pareja.

2-Actividades comunes individuales (valen un punto): el alumno/a recibe la nota obtenida de forma individual.

3-Actividades de ampliación: sirven para subir nota. Recordamos que las realiza sólo el alumnado con un ritmo de aprendizaje más rápido y son de carácter voluntario.

A la hora de otorgar el profesor/a una puntuación a cada actividad, deberá tener en cuenta la corrección de la respuesta, la ortografía, la expresión, la concreción temática, la originalidad cuando la actividad se preste a ello, etc.

Realizadas las actividades, se hace una media aritmética entre todas las puntuaciones obtenidas y se extrae así una nota final que, como se ha expuesto anteriormente, supone el $20 \%$ de la nota final del trimestre.

\section{Evaluación de la actividad}

Quizá el principal problema radica en la desigualdad de niveles aptitudinales en clase. Con el alumnado de aprendizaje más lento nos vimos obligados a trabajar de otra manera. Normalmente, 
dentro de las actividades comunes hay algunas mucho más sencillas que otras y, además, este alumnado era ayudado por otros alumnos/as de aprendizaje más rápido, con la finalidad de que trabajaran las mismas competencias y alcanzaran los mismos objetivos. Para ello, teníamos cuidado a la hora de crear las parejas o grupos, para que en todos hubiera, al menos, un alumno/a de aprendizaje rápido. Y porque no podemos olvidar que tenemos que dar respuesta a la diversidad presente en el aula (Pujolás, 2001), hemos elaborado también actividades de Ampliación, pensadas para que el alumnado de aprendizaje más rápido pueda engrandecer sus conocimientos y su aprendizaje.

Así, tras introducir actividades graduadas en complejidad podemos decir que el resultado de las mismas, encaminadas al logro de las ocho competencias básicas, es muy positivo para el alumnado de ESO, ya que, como se ha explicado con anterioridad, este asimila contenidos y destrezas relacionados con diversas áreas pero, con esta estrategia, el alumnado no nota una ruptura al cambiar de actividad y competencia sino que entiende que todas las actividades forman parte de un corpus integrado y relacionado. En palabras de Beatriz Martínez (2009, p.4), el alumnado "asimilará que el conocimiento no se puede dividir en compartimentos estancos, sino que todo se halla interrelacionado". Y todo ello se hace posible al elegir como eje de dicha batería de actividades una obra clásica de lectura obligatoria, como es el caso que nos ocupa.

\section{5.- REFERENCIAS}

Anónimo (2010). Lázaro de Tormes. Barcelona: Vicens Vives.

Chomsky, N. (1979). Reflexiones sobre el lenguaje. Barcelona: Ariel.

Coseriu, E. (1967). Sistema, norma y habla. Teoría del lenguaje y Lingüística general. Madrid: Gredos.

Estaire, S. (2004). La programación de unidades didácticas a través de las tareas. Revista RedELE, 1 [en línea]. Disponible en www.mec.es/redele/revista1/pfds1/estaire.pdf.

Gómez, A. y Arcos, D. (2007). Plan de mejora de la competencia lingüística. Elaboración del Proyecto Lingüístico de Centro. Revista de la Asociación de Inspectores de Educación en España (ADIDE), [en línea] Noviembre de 2007, Revista $n^{\circ}$ 7. Disponible en http://www.adide.org/revista (apartado de experiencias).

Habermas, J. (1987). Teoría de la acción comunicativa, Madrid: Taurus.

Jakobson, R. (1984). Ensayos de lingüística general. Barcelona: Ariel.

LOE (2006) Ley Orgánica 2/2006, de 3 de mayo, de Educación (BOE nº 106, de 4 de mayo). Madrid: Ministerio de Educación.

Martín, E. y Coll, C. (2003). Aprender contenidos, desarrollar capacidades. Barcelona: Edebé.

Martínez, B. (2009). Propuesta didáctica para fomentar la lectura y el respeto a la diversidad cultural. Espiral. Cuadernos del Profesorado [en línea], 2(4), 48-55. Disponible en:

http://www.cepcuevasolula.es/espiral.

Pujolás, P. (2001). Atención a la diversidad y aprendizaje colaborativo. Archidona: Aljibe.

Sausurre, F. (1991), Curso de Lingüística General, Madrid: Akal.

Servicio de Inspección de Las Palmas y colaboración del CEP de Telde. Materiales para el asesoramiento en Competencias Básicas [en línea]. Disponible en http://www.scribd.com/doc/23593644/DescriptoresDocumento-de-Trabajo.

Trujillo, F. (2010). Competencias básicas en el aula [en línea]. Disponible en http://prezi.com/htwhwrr7qzs8/competencias-basicas-en-el-aula.

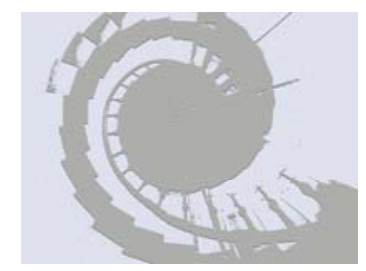

